\title{
Making Sense of a Pandemic: Mindsets Influence Emotions, Behaviors, Health, and Wellbeing During the COVID-19 Pandemic
}

\author{
Sean Zion ( $\sim$ szion@stanford.edu ) \\ Stanford University https://orcid.org/0000-0002-0802-2066 \\ Kengthsagn Louis \\ Stanford University \\ Rina Horii \\ University of Minnesota \\ Kari Leibowitz \\ Stanford University https://orcid.org/0000-0001-7494-7270

\section{Lauren Heathcote} \\ Stanford University \\ Alia Crum \\ Stanford University
}

Article

Keywords:

Posted Date: May 26th, 2021

DOI: https://doi.org/10.21203/rs.3.rs-559447/v1

License: (c) (i) This work is licensed under a Creative Commons Attribution 4.0 International License.

Read Full License

Version of Record: A version of this preprint was published at Social Science \&amp; Medicine on March 1st, 2022. See the published version at https://doi.org/10.1016/j.socscimed.2022.114889. 


\title{
Making Sense of a Pandemic: Mindsets Influence Emotions, Behaviors, Health, and Wellbeing During the COVID-19 Pandemic
}

\author{
Sean R. Zion ${ }^{1}$, Kengthsagn Louis ${ }^{1}$, Rina Horii ${ }^{2}$, Kari Leibowitz ${ }^{1}$, Lauren C. Heathcote ${ }^{3}$, Alia J. \\ Crum
}

${ }^{1}$ Stanford University Department of Psychology, Stanford, CA, USA

${ }^{2}$ University of Minnesota Department of Psychology, Minneapolis, MN, USA

${ }^{3}$ Stanford University Department of Anesthesiology, Perioperative \& Pain Medicine, Stanford, CA, USA 


\section{Abstract}

As the SARS-COV-2 virus spread across the world in the early months of 2020, people

3 sought to make sense of the complex and rapidly evolving situation. This longitudinal study of

$4 \mathrm{~N}=5,365$ Americans assessed three mindsets people formed about the COVID-19 pandemic and

5 what it meant for their lives: 'the pandemic is a catastrophe', 'the pandemic is manageable' and

6 'the pandemic can be an opportunity'. In line with our pre-registered hypotheses, these mindsets

7 were associated with a unique and largely self-fulfilling pattern of emotions (positive, negative),

8 behaviors (healthy, unhealthy, and compliance with CDC guidelines), experiences

9 (connection/growth, isolation/meaninglessness) and wellbeing (physical health, mental health,

10 quality of life). Moreover, mindsets formed in the first week of the pandemic predicted quality of

11 life 6 months later, an effect that was mediated by emotions and behaviors.

12 Keywords: COVID-19, Mindsets, Quality of Life, Health 
On March 11th, 2020, in response to the outbreak of the novel coronavirus (COVID-19),

15 the World Health Organization declared a global pandemic (World Health Organization, 2020).

16 Beset by uncertainty, people sought to make sense of this strange and unpredictable new

17 situation. Many wondered what the pandemic would mean for their own lives and for the future

18 of the world. Is this pandemic a catastrophe or is it manageable? Could it even be an opportunity

19 for societal change?

Even under normal circumstances, the world is complex and uncertain. To manage the

21 complexity and uncertainty of an ever-changing environment, we adopt mindsets — simplified

22 assumptions about the nature and workings of things in the world ${ }^{1,2}$. The assumptions we make

23 are not necessarily true or false, right or wrong. Rather, they organize and simplify complex

24 information in ways that create meaning (e.g., why is this happening?), make predictions (e.g.,

25 what will happen next?), and motivate action (e.g., what should I do?).

26 As a result, the mindsets we adopt can have a meaningful impact on our lives because

27 they shape what we feel, experience, and do ${ }^{2}$. For instance, adopting the mindset that "stress is

28 enhancing" (as opposed to "stress is debilitating") can increase positive affect, cognitive

29 flexibility, and the release of growth promoting anabolic hormones in response to a stressor ${ }^{3}$.

30 Similarly, people with more positive mindsets about the nature of aging (e.g., "aging is typified

31 by wisdom") engage in more preventative health behaviors, have fewer coronary events, and

32 even have longer lifespans than those with less adaptive mindsets (e.g., "aging is an inevitable 33 decline"') ${ }^{4}$.

34 Here we describe the extent to which people adopted the mindsets that the COVID-19

35 pandemic was 'a catastrophe,' 'manageable' or 'an opportunity'. Moreover, we explored how

36 these mindsets influenced how they felt (positive and negative affect), how they behaved 


\section{MAKING SENSE OF A PANDEMIC}

37 (healthy behaviors, unhealthy behaviors, and compliance with CDC guidelines), the types of experiences they had (isolation/meaningless, growth/connection), and how well they reported

themselves to be (physical health, mental health, and quality of life) across the first 6 months of

40 the pandemic. See Figure 1 and the measures section for additional information on the individual

41 items and the timepoints of assessment.

43 would be associated with more adaptive outcomes. People who adopted this mindset would feel

44 more positive emotions, engage in more health promoting behaviors, seek out more experiences

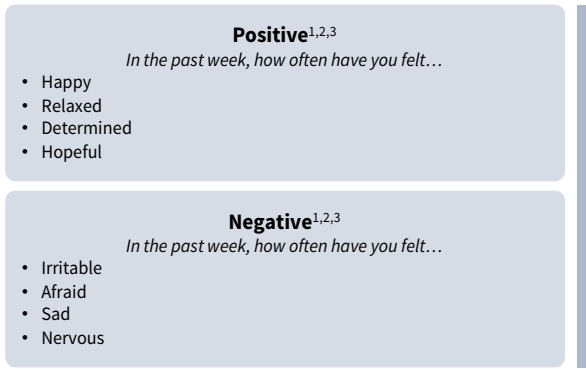

In the past week, how much have you made it a priority to...

- Avoid physical contact (at least 6 feet) with others outside your hom

- Cover your mouth and nose with a cloth or mask when around others

Stay home as much as possible

- Wash your hands

Health Promoting ${ }^{1,2,3}$

In the past week, how much have you made it a priority to.

- Get enough sleep

Exercise regularly

Unhealthy 2,3

Have you engaged in any of the following in the past month?

- I ate more pre-packaged food

I gained undesired weight

or more than desired

I drank alcohol more often than usual
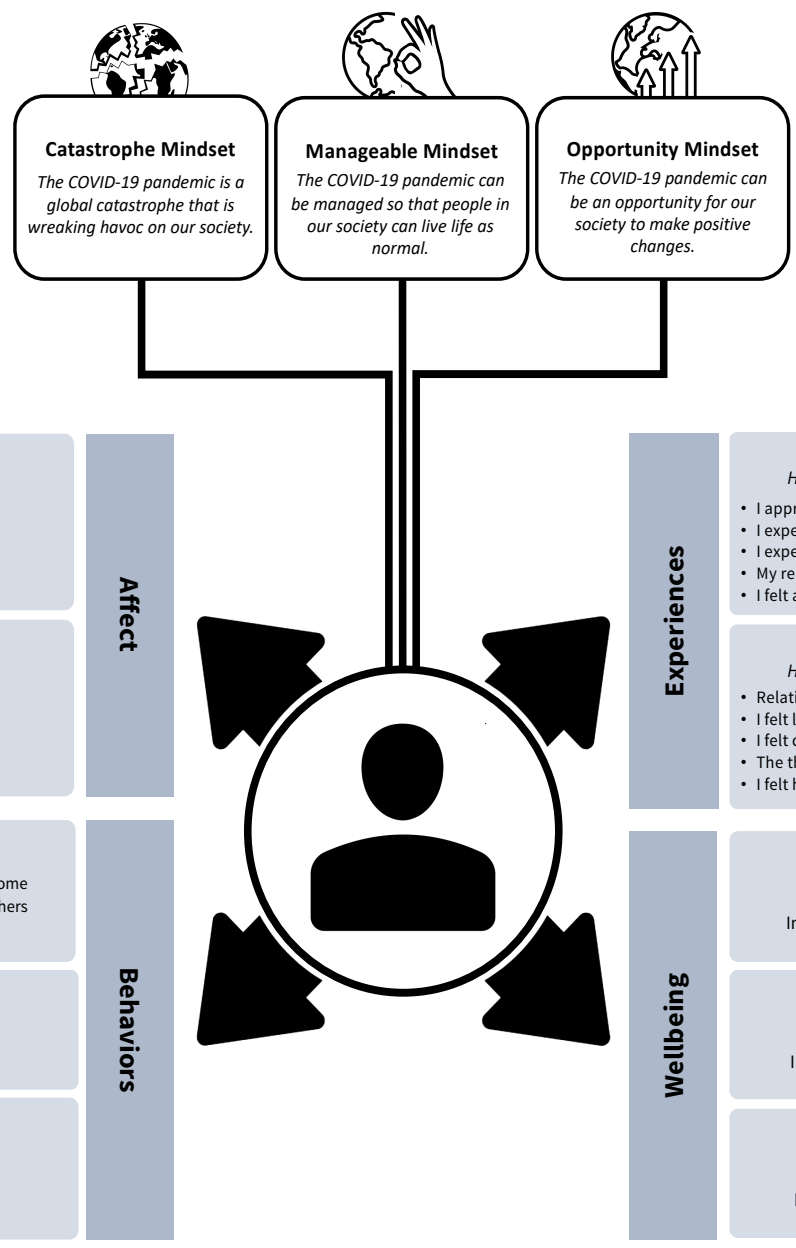

Growth \& Connection ${ }^{2,3}$ Have you experienced any of the following in the past month?

I appreciated life more

- I experienced a greater sense of connection with others - experienced personal growth/became a better version of myself - My relationship with others became stronger

- I felt a greater sense of purpose or meaning in life

Isolation \& Meaninglessness ${ }^{2,3}$

Have you experienced any of the following in the past month? - Relationships with people around me became weaker or more difficult - I felt like my life doesn't have much purpose - I felt disconnected or isolated from others

I felt hopeless about the future of the world

Physical Health ${ }^{1,2,3}$

In the past week, how would you rate your physical health?

Mental Health ${ }^{2,3}$

In the past week, how would you rate your mental health?

Quality of Life $\mathrm{L}^{1,2,3}$

In the past week, how would you rate your quality of life?

Figure 1. Outcome measures included in analyses. Superscripts indicate timepoints of assessment. T1 survey was conducted March $11^{\text {th }}-21^{\text {st }}, 2020$; T2 survey was conducted April $26^{\text {th }}-$ May $5^{\text {th }}$, 2020; T3 survey was conducted September $16^{\text {th }}-27^{\text {th }}, 2020$. 
46 we predicted that endorsement of the mindset that 'the pandemic is a catastrophe' would be

47 associated with maladaptive outcomes as the pandemic progressed. These individuals would feel

48 less positive and more negative emotions, engage in fewer health promoting and more unhealthy

49 behaviors, seek out fewer experiences of growth/connection, and report a greater number of

50 experiences of isolation/meaninglessness.

51 Our hypotheses regarding the opportunity and catastrophe mindset were largely based on

52 existing research on illness mindsets ${ }^{5}$ and stress mindsets ${ }^{6}$, however, we had relatively little

53 previous literature to go on when making predictions about the manageable mindset. On the one

54 hand, the mindset that a chronic illness is manageable is generally associated with better mental

55 and physical health ${ }^{5}$. However, in the context of a pandemic, viewing it through this lens may

56 lead people to downplay the risks of the virus and eschew the recommended safety guidelines.

57 Therefore, we hypothesized that there would be an association between this mindset and the

58 outcomes described above but did not make predictions about the direction of the associations or

59 whether the manageable mindset would be broadly adaptive or maladaptive.

For all three mindsets, we predicted relationships at the between-subjects level (e.g., on

61 average, mindsets would relate to the four categories of outcomes described above) as well as at

62 the within-subjects level (e.g., changes in an individual's endorsement of a particular mindset

63 would relate to changes in outcomes overtime). We also predicted a specific pattern of how these

64 responses would unfold overtime: mindsets formed early in the pandemic would influence

65 peoples' emotions and health related behaviors 6 weeks later, which would, in turn, influence

66 wellbeing 6 months into the pandemic.

67 Detailed hypotheses (including the directionality of the effects), our broader theoretical

68 model, a description of our measures, and our analytic approach were pre-registered on OSF 
MAKING SENSE OF A PANDEMIC

(see: https://osf.io/wufr9/).

\section{Results}

\section{Sample Demographics}

Our final sample included $\mathrm{N}=5,365$ participants who completed all three surveys.

74 Participants came from all fifty states and ranged in age from $18-89$ years (mean $=45.58, \mathrm{SD}=$

75 14.34). The sample was $81 \%$ female, $89 \%$ white, and $80 \%$ indicated educational attainment of at

76 least a high school degree. See Methods for complete details on participants and recruitment.

\section{$78 \quad$ What Mindsets Do People Endorse?}

Mean agreement with the opportunity, manageable and catastrophe mindsets at baseline

80 (T1), 6-weeks into the pandemic (T2) and 6 months into the pandemic (T3) are illustrated in

81 Figure $2 \mathrm{a}$. In the first week of the pandemic, $65.75 \%$ of people endorsed a catastrophe mindset

82 (as indicated by responding 'agree' or 'strongly agree') while only $13.77 \%$ endorsed the

83 manageable mindset. More than three quarters of participants $(76.57 \%)$ endorsed the mindset

84 that the pandemic can be an opportunity. As the pandemic progressed, the manageable mindset

85 increased whereas the catastrophe and opportunity mindsets remained relatively consistent, with

86 only slight variations occurring between timepoints. Figure 2 details the changes in mindsets as

87 well as the longitudinal changes in emotions (Figure 2b), experiences (Figure 2c), behaviors

88 (Figure 2d), and wellbeing (Figure 2e) across the three time periods collected in this study. On

89 average and in line with other research ${ }^{7}$, wellbeing declined during the first 6 months of the 90 pandemic. 


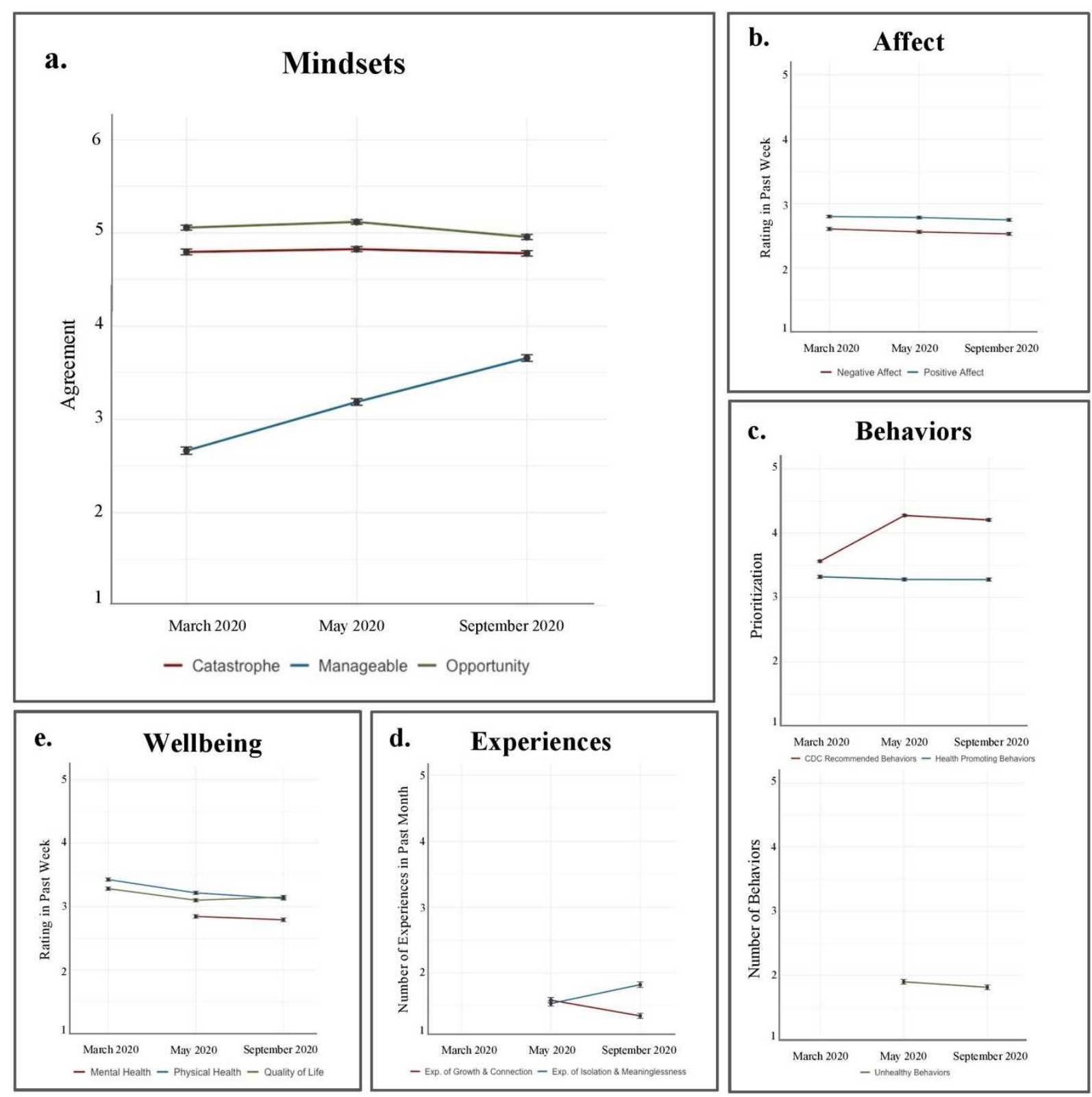

Figure 2. Changes in (a) mindsets, (b) affect, (c) behaviors, (d) experiences, and (e) wellbeing over the first six months of the COVID-19 pandemic. Error bars represent $95 \%$ confidence intervals.

92 Correlational analyses at T1 revealed that peoples' endorsement of the manageable

93 mindset and catastrophe mindset were negatively correlated $(r=-0.25 ; \mathrm{p}<0.001)$. The

94 opportunity mindset was not significantly correlated with the manageable mindset $(r=-0.03$; 
MAKING SENSE OF A PANDEMIC

$95 \mathrm{p}=0.054)$ but was positively correlated with the catastrophe mindset $(r=0.11 ; \mathrm{p}<0.001)$.

96 Correlations remained relatively stable over time (see Supplemental Table S1). None of the

97 correlations were large, which is in line with previous research suggesting these mindsets are

98 independent and not overlapping constructs ${ }^{5}$.

99 Cross sectional correlations between mindsets, emotions, experiences, behaviors, and

100 wellbeing are included in the supplemental materials (Tables S2-S4).

101

102

\section{Associations Between Mindsets and Emotions, Experiences, Behaviors, \& Wellbeing}

103

To test our pre-registered hypotheses that mindsets cause self-fulfilling changes in

104 emotions, experiences, behaviors, and wellbeing, we ran a series of mixed effects models to

105 examine between- and within- subject effects of each mindset on the outcomes listed above.

106 Between-subjects analyses explore how differences in mindsets between people relate to

107 differences in outcomes on average, collapsed over time. Within-subjects analyses provide

108 greater depth to these findings by indicating how change in an individual's mindset corresponds

109 with change in outcomes at any given time. All models controlled for age, race, gender,

110 education, and political affiliation. Due to skewness in the distribution of agreement with the

111 mindset items, these three items were log transformed prior to analysis. Complete results for both

112 between- and within- subjects effects of mindsets on outcomes are reported in Table 1.

As predicted, both between- and within- subjects analyses suggested that the 'pandemic

114 is a catastrophe' mindset was generally associated with a maladaptive pattern of emotions,

115 experiences, behaviors, and wellbeing. More specifically, between-subjects analyses revealed

116 that greater agreement with the catastrophe mindset related to less positive affect, greater

117 negative affect, more frequent unhealthy behaviors, more experiences of 
Table 1. Results of Mixed Effects Models

\begin{tabular}{|c|c|c|c|c|c|c|c|c|c|c|c|c|}
\hline \multirow[b]{3}{*}{ Emotions } & \multicolumn{4}{|c|}{ Catastrophe Mindset } & \multicolumn{4}{|c|}{ Manageable Mindset } & \multicolumn{4}{|c|}{ Opportunity Mindset } \\
\hline & \multicolumn{2}{|c|}{ Between Effects } & \multicolumn{2}{|c|}{ Within Effects } & \multicolumn{2}{|c|}{ Between Effects } & \multicolumn{2}{|c|}{ Within Effects } & \multicolumn{2}{|c|}{ Between Effects } & \multicolumn{2}{|c|}{ Within Effects } \\
\hline & Std. $\beta$ & $95 \% C I$ & Std. $\beta$ & $95 \% C I$ & Std. $\beta$ & $95 \% C I$ & Std. $\beta$ & $95 \% C I$ & Std. $\beta$ & $95 \% C I$ & Std. $\beta$ & $95 \% C I$ \\
\hline Positive & $-0.15^{\text {*** }}$ & $-0.17--0.13$ & $-0.05^{* * *}$ & $-0.06--0.04$ & $0.15^{\text {*** }}$ & $0.13-0.17$ & $0.03^{* n *}$ & $0.02-0.04$ & $0.16^{* \text { ** }}$ & $0.14-0.18$ & $0.04^{* * *}$ & $0.03-0.05$ \\
\hline Negative & $0.23^{\text {nnn }}$ & $0.21-0.25$ & $0.07^{* n *}$ & $0.06-0.08$ & $-0.14^{n * n}$ & $-0.17--0.12$ & $-0.03^{\text {nn* }}$ & $-0.04--0.02$ & -0.01 & $-0.04-0.01$ & -0.01 & $-0.02-0.00$ \\
\hline \multicolumn{13}{|l|}{ Experiences } \\
\hline Growth \& Connection & -0.01 & $-0.03-0.02$ & -0.02 & $-0.04-0.01$ & -0.03 & $-0.06-0.00$ & $-0.03^{*}$ & $-0.06--0.00$ & $0.27^{* n *}$ & $0.24-0.29$ & $0.07^{* \pm *}$ & $0.04-0.09$ \\
\hline Isolation \& Meaninglessness & $0.15^{* * *}$ & $0.13-0.18$ & 0.02 & $-0.00-0.05$ & $-0.08^{* * *}$ & $-0.12--0.06$ & 0.02 & $-0.00-0.05$ & $-0.09^{* * *}$ & $-0.12--0.06$ & $-0.04^{* *}$ & $-0.07-0.02$ \\
\hline \multicolumn{13}{|l|}{ Behaviors } \\
\hline Unhealthy Behaviors & $0.12^{* * \star}$ & $0.10-0.15$ & $0.05^{* \star *}$ & $0.02-0.07$ & $-0.07^{* * *}$ & $-0.09--0.04$ & 0.03 & $0.00-0.05$ & -0.02 & $-0.05-0.00$ & 0.00 & $-0.02-0.03$ \\
\hline Healthy Behaviors & -0.02 & $-0.04-0.00$ & -0.01 & $-0.02-0.00$ & $0.04^{* *}$ & $0.02-0.06$ & 0.01 & $-0.00-0.02$ & $0.11^{* * *}$ & $0.09-0.13$ & $0.02^{n+n}$ & $0.01-0.03$ \\
\hline CDC Behaviors & $0.21^{* * *}$ & $0.10-0.23$ & $0.05^{\star * *}$ & $0.04-0.06$ & $-0.23^{* * *}$ & $-0.25--0.21$ & $-0.05^{\text {*** }}$ & $-0.06--0.04$ & $0.19^{* * n}$ & $0.17-0.21$ & $0.04^{* * *}$ & $0.03-0.05$ \\
\hline \multicolumn{13}{|l|}{ Health \& Wellbeing } \\
\hline Physical Health & $-0.08^{n \hbar \hbar}$ & $-0.10--0.06$ & $-0.02^{n+n}$ & $-0.03--0.01$ & $0.13^{n+*}$ & $0.10-0.15$ & 0.01 & $-0.00-0.02$ & $0.03^{*}$ & $0.01-0.05$ & 0.01 & $0.00-0.03$ \\
\hline Mental Health & $-0.14^{n * \hbar}$ & $-0.17--0.12$ & -0.01 & $-0.04-0.01$ & $0.10^{n * n}$ & $0.08-0.13$ & -0.02 & $-0.04-0.01$ & $0.07^{n+n}$ & $0.04-0.10$ & $0.04^{n \star}$ & $0.01-0.06$ \\
\hline Quality of Life & $-0.14^{* * *}$ & $-0.16--0.12$ & $-0.05^{\text {*** }}$ & $-0.06--0.04$ & $0.11^{* * * *}$ & $0.09-0.14$ & 0.01 & $-0.01-0.02$ & $0.12^{\star * * *}$ & $0.10-0.14$ & $0.03^{* n *}$ & $0.02-0.04$ \\
\hline
\end{tabular}

Table 1. Results of mixed effects models. Standardized betas and $95 \%$ CI are reported for between-subjects effects and within-subjects effects for each of the three mindsets. Mindsets were log transformed prior to analyses. All mixed effects models controlled for age, race, gender, education attainment, and political affiliation. Asterisks indicate level of significance such that ${ }^{*} p \leq 0.05 ;{ }^{* *} p \leq \mathbf{0 . 0 1} ;{ }^{* * *} \boldsymbol{p} \leq \mathbf{0 . 0 0 1}$. Significant findings at the $\mathrm{p} \leq 0.01$ level are bolded.

118 isolation/meaninglessness, worse physical/mental health, and lower quality of life. Within-

119 subjects analyses mirrored these patterns, suggesting that an increase in agreement with the

120 catastrophe mindset was associated with a corresponding decrease in positive affect, physical

121 health, and quality of life, and an increase in negative affect, and unhealthy behaviors. Counter to

122 our predictions, we did not observe a significant association between the catastrophe mindset and

123 experiences of growth/connection, nor were changes in the catastrophe mindset associated with

124 corresponding changes in experiences of isolation/meaninglessness or mental health.

125 Also as predicted, the mindset that 'the pandemic is an opportunity' was associated with

126 an adaptive pattern of emotions, experiences, behaviors, and wellbeing. Between-subjects

127 analyses indicated that greater agreement with the opportunity mindset was associated with 
129 growth/connection, better mental health, and better quality of life. Within-subjects analyses 130 mirrored these results in all cases, although the associations were weaker in magnitude. Counter

131 to our predictions, the opportunity mindset was not significantly associated with a reduction in 132 unhealthy behaviors, and the effect on physical health was negligible (and not significant at the $133 \mathrm{p}<.01$ level). As predicted, the opportunity mindset was not associated with negative affect. Exploratory analyses of the mindset that 'the pandemic is manageable' suggested that, 135 between subjects, higher endorsement of this mindset was associated with greater positive affect, 136 less negative affect, fewer experiences of isolation/meaninglessness, more healthy behaviors, 137 fewer unhealthy behaviors, and better wellbeing (physical health, mental health, and quality of 138 life). Only the association with affect was significant $(\mathrm{p}<0.001)$ at the within-subjects level. Exploratory analyses of the association between mindsets and compliance with CDC 140 guidelines suggested that both the catastrophe and the opportunity mindset were significantly

141 associated with greater compliance with CDC recommended behaviors. Conversely, we observed 142 significant negative between- and within- subjects effects of the manageable mindset on 143 compliance with CDC recommended behaviors.

145 How do Mindsets at the Start of the Pandemic Impact Quality of Life 6 Months Later?

146 To test our theoretical model that mindsets lead to differences in affect and behavior, 147 which, in turn, influence more global assessments of health and wellbeing, we explored three 148 multiple mediation models. Each model included the mindset measured at T1 as the independent 149 variable and quality of life measured at T3 as the outcome variable. Four mediators, each 150 measured at T2, were included: negative affect, positive affect, healthy behaviors, and unhealthy 151 behaviors. Engagement in CDC recommended behaviors were not included in mediation models 
152 as we did not have a clear theoretical rationale for how they would mediate the effects of the 153 mindsets on quality of life in cases where COVID-19 was not contracted. Experiences of

154 growth/connection and isolation/meaninglessness were also not included in mediation models as 155 they were not a part of our pre-registered theoretical model. All mediation models controlled for 156 age, race, gender, education, and political affiliation. Due to skewness in the distribution of 157 agreement with the mindset items, these three items were log transformed prior to analysis. Results of these mediation models are reported in Figure 3. The relationship between the

159 catastrophe mindset in March 2020 and quality of life six months later was mediated by 160 increased negative affect, decreased positive affect, and engagement in unhealthy behaviors 161 reported in early May (Figure 3a). The pandemic opportunity mindset influenced quality of life

162 six months later through its impact on positive affect and prioritization of health promoting 163 behaviors (Figure 3b). Finally, the pandemic manageable mindset shaped quality of life through 164 affective - but not behavioral - mediators (Figure 3c). Similar effects were noted for mental and 165 physical health outcomes (See Supplement Figure S2)

\section{Discussion}

While the number of COVID-19 infections ebbed and flowed over the course of 2020, for

168 many, the distress of the pandemic itself only compounded with time. However, there was a 169 great deal of heterogeneity in the lived experiences of people during this time. This longitudinal 170 study of 5,365 Americans provides insight into how three mindsets about the pandemic - that it is 171 'a catastrophe', 'manageable', or 'an opportunity' - explain some of this heterogeneity through 
172 their self-fulfilling impact on peoples' emotions, behaviors, health, and wellbeing.
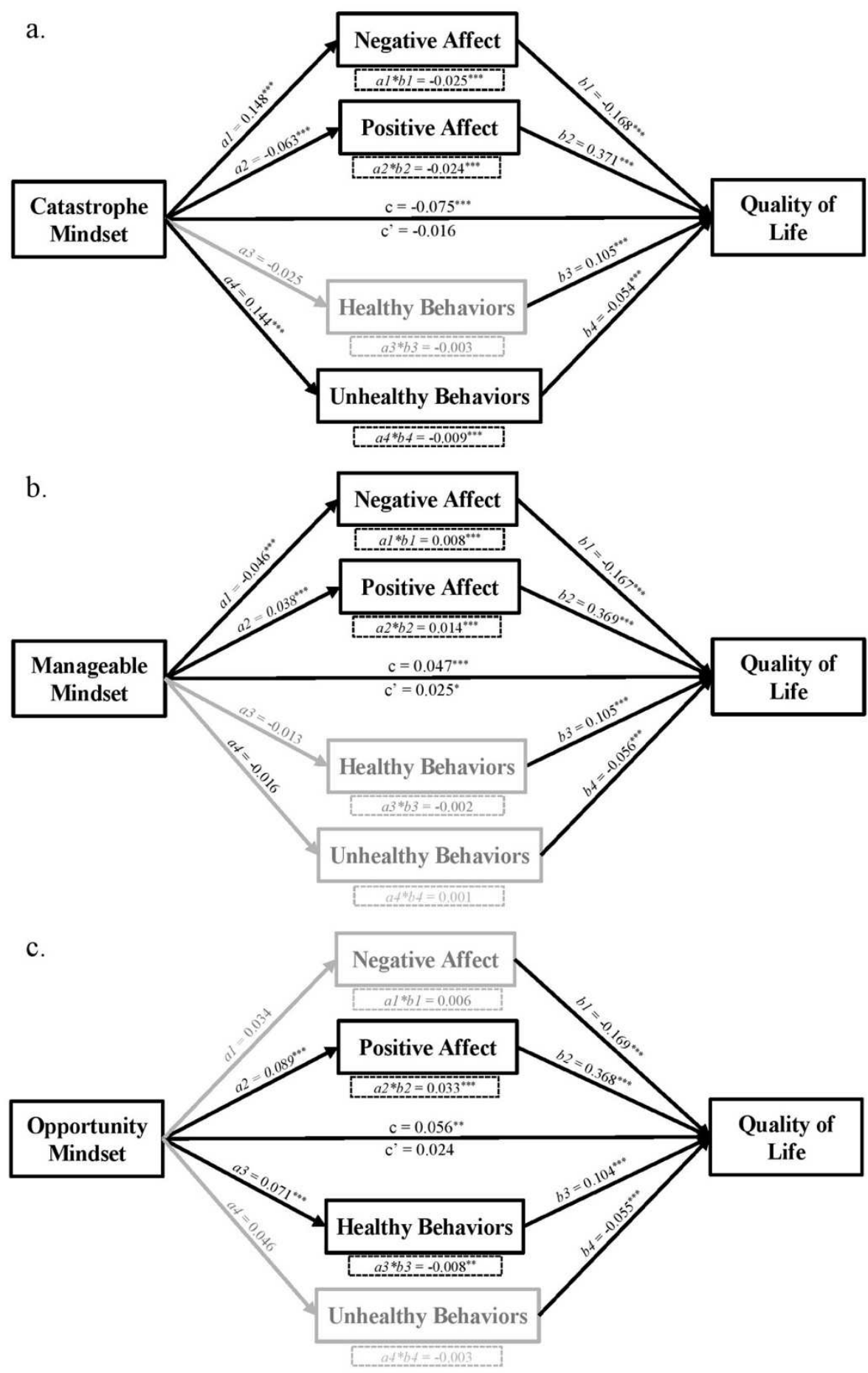

Figure 3. Mediation models outlining the mechanisms through which the (a) catastrophe mindset, (b) manageable mindset, and (c) opportunity mindset influence quality of life. Mindsets were measured at T1, affective and behavioral mediators were measured at T2, and quality of life was measured at T3. Standardized estimates of direct and indirect effects are listed. Non-significant effects are indicated by faded text and gray arrows. Asterisks indicate level of significant such that ${ }^{*} p \leq 0.05 ;{ }^{* *} p \leq 0.01 ;{ }^{* * *} p \leq 0.001$. 
We assessed mindsets about the COVID-19 pandemic at three timepoints: March, May, 174 and September of 2020. At the start of the pandemic, the catastrophe and opportunity mindsets

175 were both widely adopted, however they oriented people to two very different realities. Those 176 who held the mindset that the pandemic was a catastrophe were more likely to experience higher

177 levels of negative affect, lower levels of positive affect, greater engagement in unhealthy

178 behaviors, more frequent experiences of isolation/meaninglessness, and report worse wellbeing.

179 Conversely, agreement with the opportunity mindset related to greater positive affect, more

180 experiences of growth/connection, fewer experiences of isolation/meaninglessness, and better

181 wellbeing. Relatively few people initially adopted the mindset that the COVID-19 pandemic was

182 manageable; however, agreement with this mindset increased steadily over time. The

183 manageable mindset related to several individually adaptive outcomes, including engagement in

184 fewer unhealthy behaviors, greater positive affect, less negative affect, and greater wellbeing;

185 however, it also strongly predicted lower prioritization of CDC recommended behaviors.

The between- and within-subjects effects of mindsets on affect, behavior, experiences,

187 and wellbeing suggest that mindsets about the pandemic influenced people in self-fulfilling

188 ways. The longitudinal multiple mediation analyses add to this finding by demonstrating a

189 potential mechanism through which some of these effects operate. We found that mindsets at the

190 start of the pandemic predicted quality of life 6 months later through their impact on affective

191 and behavioral processes. This supports our theoretical model that mindsets influence wellbeing

192 outcomes through affective and behavioral pathways; in particular, different mindsets shape

193 these mediators in slightly different ways, which is in line with existing research on stress

194 mindsets ${ }^{3}$. 
MAKING SENSE OF A PANDEMIC

While the mindsets people adopted in response to the COVID-19 pandemic were impactful, they were not necessarily a full or accurate reflection of reality. The data collected

197 during the first week of the pandemic make this clear. People were likely reporting their 198 mindsets about the pandemic before they fully understood the threat posed by the virus or experienced the true impact of the pandemic on their daily lives. In other words, people were not

200 necessarily reporting that the pandemic was a catastrophe, manageable, or an opportunity

201 because that was a reflection of their actual experience with the pandemic. But the way we 202 organize and simplify complex information can critically shape our lives by operating in self203 fulfilling ways. This was demonstrated by the fact that these early mindsets predicted important 204 outcomes 6 months later.

That said, just because mindsets are not a direct reflection of reality does not mean they are a rejection of reality. A novel virus was rapidly spreading around the globe and people had to

207 make sense of the evolving situation and plan a course of action. The data from this study clearly 208 indicate that mindsets shaped COVID-19 relevant behaviors people engaged in. Those who 209 viewed the pandemic through the lens of the catastrophe mindset took the situation more 210 seriously; they stayed home, washed their hands, and (when it was recommended) started

211 wearing a mask. Interestingly, this appeared to be at the expense of other aspects of their

212 wellbeing. This contrasts with those who adopted the manageable mindset. Despite maintaining

213 high levels of wellbeing during the pandemic, people who adopted this mindset were much less

214 likely to prioritize these CDC recommendations. As such, endorsement of this mindset may 215 reflect an attempt to deny the reality of the global pandemic and a refusal to engage with it in a 216 socially responsible way. Over time, as people adjusted to the changes necessitated by the 217 pandemic and it may have become more adaptive. 
MAKING SENSE OF A PANDEMIC

The opportunity mindset seemed to provide the best of both worldviews; those who

219 adopted this mindset staved off major declines in wellbeing without subverting the behaviors

220 necessary to engage with the pandemic in a socially responsible way. Interestingly, however, this

221 mindset did not appear to boost self-reported physical health or reduce negative affect. The latter

222 effect is consistent with research on stress mindsets, which suggests a stress-can-be-enhancing

223 mindset - similar to the mindset that the pandemic can be an opportunity - relates to increased

224 positive affect but does not necessarily drive people to avoid negative experiences ${ }^{3}$.

225 Contrary to previous research on the role of these mindsets in chronic illness ${ }^{5}$, we

226 observed a positive correlation between the catastrophe and opportunity mindsets in this context.

227 What do we make of this? Perhaps some acknowledgement of a situation as a catastrophe is

228 necessary to recognize it as an opportunity. Perhaps a more useful mindset to explore in future

229 research is something to the accord of "catastrophes can present opportunities for positive

230 change'. Indeed, people can likely hold multiple, even opposing, mindsets simultaneously.

231 Broadly, it suggests that seeing this pandemic as an opportunity does not necessarily require an

232 individual to overlook or deny the negative aspects of this unique and challenging situation. This

233 is one of the benefits of mindsets: they help us simplify and organize information about a

234 complex concept while allowing room for more nuance than a good vs. bad or right vs. wrong

235 judgement.

There are several limitations of this study that should be considered when interpreting the

237 results. First, this sample is not representative of the United States as a whole. Therefore, we do 238 not intend to make claims about mindsets at a population level, but rather to explore the

239 relationships between mindsets and important outcomes within this sample. Relatedly, our

240 sample is disproportionately female, white, and educated. This is likely due to our social media- 
241 based recruitment strategy. Advertisements on social media platforms are selectively displayed

242 to individuals deemed likely to engage with the content. While this allowed us to quickly survey

243 a large sample of participants, it came at the cost of a truly representative sample in terms of

244 gender, race, and socioeconomic status. Additional research is needed to explore whether these

245 patterns hold in more diverse/representative samples.

246 Second, we lost to many participants to retention over the course of this 6-month long

247 study. This is likely due to our recruitment strategy, the method of follow-up (e.g., via email),

248 and the unique individual experiences of participants during the pandemic that took priority over

249 completing our follow-up survey. Analyses of baseline variables across retention rate suggest

250 that differences in demographic variables, individual differences, mindsets, and outcomes, were

251 significant at the $\mathrm{p}<0.05$ level, but not large in magnitude. We have included additional details

252 on these differences in the supplemental materials.

253 Third, we acknowledge that mindsets are just one piece of the puzzle. We do not wish to

254 discount the many structural or situational variables (e.g., access and affordability of healthcare)

255 that can impact health and wellbeing during a pandemic. We also do not wish to suggest that

256 mindsets are a substitute for proper mental health care for those who need it during this

257 challenging time.

258 Fourth, we aim to describe these mindsets, not prescribe them. While some mindsets may

259 be more useful than others for specific goals, there isn't necessarily a right or wrong mindset for

260 people to adopt as they navigate this pandemic. For example, the mindset that the pandemic is a

261 catastrophe may certainly be maladaptive in many ways, but it appears to predict higher levels of

262 engagement with CDC recommended behaviors. That said, this study hints at mindsets that could 
263 be more adaptive than others and future research may explore intervention strategies that help 264 people adopt mindsets that best serve their individual goals.

265 Just as SARS-CoV-2 mutates and evolves over time, the lived experience of people

266 around the world continues to change in response to the global pandemic. Although much

267 remains to be explored - and cumulative effects of a distressing year have yet to be fully

268 understood - this work provides insight into how people's mindsets shaped their wellbeing

269 during the COVID-19 pandemic. It may also help to inform best practices for public messaging

270 or targeted interventions for future societal disruptions. As vaccinations roll out and to millions

271 around the world, there appears to be a light at the end of this tunnel. How will our mindsets

272 affect the meaning we assign to this year spent in relative isolation from friends, family, and the

273 normal rhythms of life? And perhaps more importantly, if our mindsets shaped our lived

274 experiences during the pandemic, how might they relate to our wellbeing in a post-pandemic

275 world?

\section{Methods}

\section{Participants and Procedure}

American adults were recruited to participate in a survey about their perceptions of and

279 reactions to the COVID-19 pandemic through a social media advertising campaign on Facebook 280 and Twitter (See Figure S1 in the Supplementary Materials). All U.S. residents over the age of

28118 were eligible to participate in the study. Recruitment for the first survey (T1) took place over

282 a ten-day period starting on March $11^{\text {th }}, 2020$, the day the World Health Organization (WHO)

283 officially declared COVID-19 a global pandemic. Data was collected from 22,276 participants at

$284 \mathrm{~T} 1,16,327$ of whom met inclusion and data quality criteria and provided an email address as 
MAKING SENSE OF A PANDEMIC

285

286

287

288

289

290

291

292

293

294

295

296

297

298

299

300

301

302

303

304

305

306

307

consent to be contacted for follow-up survey.

Participants were invited over email to complete two follow-up surveys approximately 6weeks (T2; May 2020) and 6-months (T3; October 2020) after the initial survey. N=9,643 participants completed the second survey and $\mathrm{N}=7,287$ participants completed the third survey. Participants who indicated contracting COVID-19 $(\mathrm{N}=72)$ were removed prior to analyses. A total of $\mathrm{N}=5,365$ COVID-negative participants completed all three surveys and were included in the subsequent longitudinal analyses. Within this sample of $\mathrm{N}=5,365$ participants, items that were missing at random were imputed using multiple imputation methods (e.g., predictive mean matching) in R using the MICE package, which can be valid and unbiased methods for data that is missing at random ${ }^{8}$. Responses were imputed for 84 participants who had one or more missing responses determined to be missing at random. Baseline differences in demographics, mindsets, and outcome variables across retention rate are included in supplemental tables S5-S7.

\section{Measures}

Mindsets. Mindsets about the COVID-19 Pandemic were measured using an adapted version of the Illness Mindset Inventory (IMI), which measures 3 mindsets about the nature and meaning of illness: that it is a catastrophe, manageable, or an opportunity ${ }^{5}$. Mindsets were assessed with a single item rated on a 6-point Likert scale ranging from (1) strongly disagree to (6) strongly agree.

Emotions. Emotions were measured using an adapted version of the Positive and Negative Affect Scale (PANAS) that asked participants to indicate the extent to which they have felt four positive emotions (happy, relaxed, determined, hopeful) and four negative emotions (irritable, afraid, sad, nervous) over the last week ${ }^{9}$. Separate summary scores were calculated for 
MAKING SENSE OF A PANDEMIC

308 positive affect (alpha $=0.75-0.79$ across timepoints $)$ and negative affect (alpha $=0.73-0.82$

309 across timepoints) by averaging the respective items at each timepoint.

Health Behaviors. Engagement of CDC recommended behaviors was measured by

311 asking how much participants prioritized physical distancing, staying home, wearing a face

312 mask, and hand washing. Summary scores were calculated by averaging the items at each

313 timepoint (alpha $=0.53-0.74$ across timepoints).

Health promoting behaviors were measured by asking how much participants prioritized

315 getting enough sleep, eating nutritiously, and exercising regularly. Summary scores were

316 calculated by averaging the items at each timepoint (alpha $=0.68-0.71$ across timepoints).

Unhealthy behaviors were measured using a binary (i.e., yes or no) checklist of items

318 related to eating more pre-packaged food, gaining undesired weight, and sleeping irregularly. A

319 total score for unhealthy behaviors was calculated at each timepoint by taking the sum of the 320 three items.

Experiences. We measured two categories of experiences: experiences characterized by

322 isolation or meaninglessness (e.g., the things I do felt meaningless; I felt hopeless about the

323 future of the world), and experiences characterized by personal growth or connection (e.g. I

324 appreciated life more; I felt a greater sense of purpose or meaning in life). Each category

325 consisted of five items assessed over the previous month using a binary (i.e., yes or no) checklist.

326 Total scores for both categories of experiences were calculated by taking the sum of the

327 respective items at each timepoint.

Wellbeing. Mental health, physical health, and quality of life were each measured using a

329 single item from the PROMIS Global Health Scale version $1.2^{10}$. 
MAKING SENSE OF A PANDEMIC

\section{References}

331 1. Molden, D. C. \& Dweck, C. S. Finding 'meaning' in psychology: A lay theories approach 332 to self-regulation, social perception, and social development. Am. Psychol. 61, 192-203 $333 \quad$ (2006).

334 2. Crum, A. J. Mindsets and Health. (2021).

335 3. Crum, A. J., Akinola, M., Martin, A. \& Fath, S. The role of stress mindset in shaping 336 cognitive, emotional, and physiological responses to challenging and threatening stress. Anxiety, Stress. Coping 1-17 (2017). doi:10.1080/10615806.2016.1275585

338 4. Levy, B. Stereotype embodiment: A psychosocial approach to aging. Curr. Dir. Psychol. 339 Sci. (2009). doi:10.1111/j.1467-8721.2009.01662.x

340 5. Zion, S. R. et al. Illness Mindsets in Health and Disease: Development \& Validation of 341 the Illness Mindset Inventory (IMI). (2021).

342 6. Crum, A. J., Salovey, P. \& Achor, S. Rethinking stress: The role of mindsets in 343 determining the stress response. J. Pers. Soc. Psychol. 104, 716-733 (2013).

344 7. Zacher, H. \& Rudolph, C. W. Individual differences and changes in subjective wellbeing 345 during the early stages of the COVID-19 pandemic. Am. Psychol. 76, 50-62 (2021).

346 8. Bell, M. L., Fiero, M., Horton, N. J. \& Hsu, C. H. Handling missing data in RCTs; A 347 review of the top medical journals. BMC Med. Res. Methodol. 14, 1-8 (2014).

348 9. Crawford, J. R. \& Henry, J. D. The Positive and Negative Affect Schedule (PANAS): 349 Construct validity, measurement properties and normative data in a large non-clinical 350 sample. Br. J. Clin. Psychol. 43, 245-265 (2004). 
MAKING SENSE OF A PANDEMIC

351 10. Cella, D. et al. The Patient-Reported Outcomes Measurement Information System 


\section{Supplementary Materials}

Table S1. Correlations between mindsets at T1, T2, and T3

\begin{tabular}{lcccccccccc}
\hline & $\boldsymbol{M}$ & $\boldsymbol{S D}$ & $\mathbf{1}$ & $\mathbf{2}$ & $\mathbf{3}$ & $\mathbf{4}$ & $\mathbf{5}$ & $\mathbf{6}$ & $\mathbf{7}$ & $\boldsymbol{8}$ \\
\hline Mindsets at T1 & & & & & & & & & & \\
1. Catastrophe Mindset & 4.80 & 1.17 & & & & & & & \\
2. Manageable Mindset & 2.67 & 1.46 & $-.25^{* *}$ & & & & & & \\
3. Opportunity Mindset & 5.06 & 0.99 & $.11^{* *}$ & -.03 & & & & & \\
\hline Mindsets at T2 & & & & & & & & & \\
4. Catastrophe Mindset & 4.83 & 1.02 & $.36^{* *}$ & $-.16^{* *}$ & $.05^{* *}$ & & & & \\
5. Manageable Mindset & 3.19 & 1.34 & $-.15^{* *}$ & $.44^{* *}$ & $-.08^{* *}$ & $-.20^{* *}$ & & & \\
6. Opportunity Mindset & 5.12 & 0.92 & $.07^{* *}$ & $-.10^{* *}$ & $.45^{* *}$ & $.10^{* *}$ & $-.14^{* *}$ & & & \\
\hline Mindsets at T3 & & & & & & & & & \\
7. Catastrophe Mindset & 4.78 & 1.08 & $.31^{* *}$ & $-.15^{* *}$ & $.10^{* *}$ & $.41^{* *}$ & $-.21^{* *}$ & $.11^{* *}$ & & \\
8. Manageable Mindset & 3.66 & 1.34 & $-.12^{* *}$ & $.34^{* *}$ & $-.05^{* *}$ & $-.14^{* *}$ & $.48^{* *}$ & $-.09^{* *}$ & $-.17^{* *}$ & \\
9. Opportunity Mindset & 4.96 & 1.02 & $.08^{* *}$ & $-.09^{* *}$ & $.41^{* *}$ & $.09^{* *}$ & $-.16^{* *}$ & $.53^{* *}$ & $.20^{* *}$ & $-.07^{* *}$ \\
\hline
\end{tabular}

Table S1. Correlations between mindsets measured at T1 (March 2020), T2 (May 2020), and T3 (September 2020). M and SD indicate mean and standard deviation, respectively. Asterisks indicate significance of Pearson's correlations such that * $\mathrm{p} \leq 0.01 ;{ }^{* *} \mathrm{p} \leq 0.001$. 
Table S2. Correlations between measures at T1

\begin{tabular}{|c|c|c|c|c|c|c|c|c|c|c|c|}
\hline Variables at $\mathbf{T 1}$ & $M$ & $S D$ & 1 & 2 & 3 & 4 & 5 & 6 & 7 & 8 & 9 \\
\hline \multicolumn{12}{|l|}{ Affect } \\
\hline 1. Positive Affect & 2.80 & 0.77 & $(0.74)$ & & & & & & & & \\
\hline 2. Negative Affect & 2.61 & 0.92 & $-.51^{* *}$ & $(0.72)$ & & & & & & & \\
\hline \multicolumn{12}{|l|}{ Behaviors } \\
\hline 3. CDC Recommendations & 3.56 & 0.55 & -.02 & $.17^{* *}$ & - & & & & & & \\
\hline 4. Healthy & 3.32 & 0.86 & $.28^{* *}$ & $-.14^{* *}$ & $.20^{* *}$ & $(0.71)$ & & & & & \\
\hline \multicolumn{12}{|l|}{ Wellbeing } \\
\hline 5. Quality of Life & 3.28 & 0.99 & $.52^{* *}$ & $-.47^{* *}$ & $-.04^{* *}$ & $.22^{* *}$ & - & & & & \\
\hline 6. Phys. Health & 3.42 & 0.97 & $.32^{* *}$ & $-.24^{* *}$ & $-.07^{* *}$ & $.28^{* *}$ & $.47^{* *}$ & - & & & \\
\hline \multicolumn{12}{|l|}{ Mindsets } \\
\hline 7. Catastrophe & 4.80 & 1.17 & $-.17^{* *}$ & $.27^{* *}$ & $.25^{* *}$ & .00 & $-.15^{* *}$ & $-.07^{* *}$ & - & & \\
\hline 8. Manageable & 2.67 & 1.46 & $.14^{* *}$ & $-.13^{* *}$ & $-.23^{* *}$ & -.03 & $.07^{* *}$ & $.08^{* *}$ & $-.25^{* *}$ & - & \\
\hline 9. Opportunity & 5.06 & 0.99 & $.11^{* *}$ & .02 & $.15^{* *}$ & $.09^{* *}$ & $.08^{* *}$ & .00 & $.11^{* *}$ & -.03 & - \\
\hline
\end{tabular}

Table S2. Correlations between measures of affect, behaviors, wellbeing, and mindsets measured at T1 (March $11^{\text {th }}-21^{\text {st }}, 2020$ ). $\mathrm{M}$ and SD indicate mean and standard deviation, respectively. Asterisks indicate significance of Pearson's correlations such that ${ }^{*} \mathrm{p} \leq 0.01$ and ${ }^{* *} \mathrm{p} \leq 0.001$. Cronbach's alphas are included in parentheses on the diagonal for summary scores (but not total scores or individual items). 
Table S3. Correlations between measures at T2

\begin{tabular}{|c|c|c|c|c|c|c|c|c|c|c|c|c|c|c|c|}
\hline Variables at $\mathrm{T} 2$ & $M$ & $S D$ & 1 & 2 & 3 & 4 & 5 & 6 & 7 & 8 & 9 & 10 & 11 & 12 & 13 \\
\hline \multicolumn{16}{|l|}{ Affect } \\
\hline 1. Positive & 2.78 & 0.76 & $(0.80)$ & & & & & & & & & & & & \\
\hline 2. Negative & 2.56 & 0.89 & $-.53^{* *}$ & $(0.77)$ & & & & & & & & & & & \\
\hline \multicolumn{16}{|l|}{ Behaviors } \\
\hline 3. CDC Recommendations & 4.27 & 0.64 & .04 & $.12^{* *}$ & - & & & & & & & & & & \\
\hline 4. Healthy & 3.28 & 0.87 & $.36^{* *}$ & $-.23^{* *}$ & $.15^{* *}($ & $(0.68)$ & & & & & & & & & \\
\hline 5. Unhealthy & 1.90 & 1.33 & $-.34^{* *}$ & $.40^{* *}$ & $.05^{* *}$ & $-.43^{* *}$ & - & & & & & & & & \\
\hline \multicolumn{16}{|l|}{ Experiences } \\
\hline 6. Growth/Connection & 1.59 & 1.48 & $.39^{* *}$ & $-.12^{* *}$ & $.16^{* *}$ & $.22^{* *}$ & $-.06^{* *}$ & & & & & & & & \\
\hline 7. Isolation/Meaninglessness & 1.54 & 1.42 & $-.47^{* *}$ & $.57^{* *}$ & -.02 & $-.23^{* *}$ & $.39^{* *}$ & $-.18^{* *}$ & - & & & & & & \\
\hline \multicolumn{16}{|c|}{ Wellbeing } \\
\hline 8. Quality of Life & 3.10 & 0.93 & $.56^{* *}$ & $-.48^{* *}$ & .00 & $.31^{* *}$ & $-.34^{* *}$ & $.30^{* *}$ & $-.45^{* *}$ & - & & & & & \\
\hline 9. Physical Health & 3.22 & 0.93 & $.34^{* *}$ & $-.29^{* *}$ & $-.07^{* *}$ & $.39^{* *}$ & $-.34^{* *}$ & $.15^{* *}$ & $-.23^{* *}$ & $.50^{* *}$ & - & & & & \\
\hline 10. Mental Health & 2.84 & 1.04 & $.63^{* *}$ & $-.67^{* *}$ & $-.04^{*}$ & $.33^{* *}$ & $-.41^{* *}$ & $.23^{* *}$ & $-.55^{* *}$ & $.66^{* *}$ & $.45^{* *}$ & - & & & \\
\hline \multicolumn{16}{|l|}{ Mindsets } \\
\hline 11. Catastrophe & 4.83 & 1.02 & $-.15^{* *}$ & $.21^{* *}$ & $.21^{* *}$ & -.02 & $.13^{* *}$ & -.01 & $.15^{* *}$ & $-.12^{* *}$ & $-.06^{* *}$ & $-.13^{* *}$ & - & & \\
\hline 12. Manageable & 3.19 & 1.34 & $.12^{* *}$ & $-.13^{* *}$ & $-.29^{* *}$ & -.01 & $-.05^{* *}$ & $-.06^{* *}$ & $-.08^{* *}$ & $.04^{*}$ & $.09^{* *}$ & $.09^{* *}$ & $-.20^{* *}$ & - & \\
\hline 13. Opportunity & 5.12 & 0.92 & $.13^{* *}$ & .01 & $.21^{* *}$ & $.09^{* *}$ & .03 & $.25^{* *}$ & $-.04^{*}$ & $.13^{* *}$ & .00 & .03 & $.10^{* *}$ & $-.14^{* *}$ & - \\
\hline
\end{tabular}

Table S3. Correlations between measures of affect, behaviors, wellbeing, and mindsets measured at T2 (April $26^{\text {th }}-$ May $5^{\text {th }}, 2020$ ). M and SD indicate mean and standard deviation, respectively. Asterisks indicate significance of Pearson's correlations such that ${ }^{*} \mathrm{p} \leq 0.01$ and ${ }^{* *} \mathrm{p} \leq 0.001$. Cronbach's alphas are included in parentheses on the diagonal for summary scores (but not total scores or individual items). 
Table S4. Correlations between measures at T3

\begin{tabular}{|c|c|c|c|c|c|c|c|c|c|c|c|c|c|c|}
\hline Variables at T3 & $M$ & $S D$ & 1 & 2 & 3 & 4 & 5 & 6 & 7 & 8 & 9 & 10 & 11 & 1213 \\
\hline \multicolumn{15}{|l|}{ Affect } \\
\hline 1. Positive & 2.75 & 0.79 & $(0.79)$ & & & & & & & & & & & \\
\hline 2. Negative & 2.53 & 0.87 & $-.53^{* * *}$ & $(0.82)$ & & & & & & & & & & \\
\hline \multicolumn{15}{|l|}{ Behaviors } \\
\hline 3. CDC Recommendations & 4.20 & 0.73 & $-.08^{* *}$ & $.19^{* *}$ & $(0.74)$ & & & & & & & & & \\
\hline 4. Healthy & 3.28 & 0.91 & $.38^{* *}$ & $-.26^{* *}$ & $.10^{* *}$ & $(0.71)$ & & & & & & & & \\
\hline 5. Unhealthy & 1.81 & 1.36 & $-.35^{* *}$ & $.42^{* *}$ & $.13^{* *}$ & $-.41^{* *}$ & - & & & & & & & \\
\hline \multicolumn{15}{|l|}{ Experiences } \\
\hline 6. Growth/Connection & 1.35 & 1.44 & $.41^{* *}$ & $-.13^{* *}$ & $.10^{* *}$ & $.22^{* *}$ & $-.06^{* *}$ & - & & & & & & \\
\hline 7. Isolation/Meaninglessness & 1.82 & 1.55 & $-.52^{* *}$ & $.59^{* *}$ & $.11^{* *}$ & $-.24^{* *}$ & $.42^{* *}$ & $-.20^{* *}$ & - & & & & & \\
\hline \multicolumn{15}{|l|}{ Wellbeing } \\
\hline 8. Quality of Life & 3.15 & 0.96 & $.61^{* *}$ & $-.47^{* *}$ & $-.09^{* *}$ & $.35^{* *}$ & $-.36^{* *}$ & $.27^{* *}$ & $-.47^{* *}$ & - & & & & \\
\hline 9. Physical Health & 3.13 & 0.94 & $.44^{* *}$ & $-.34^{* *}$ & $-.11^{* *}$ & $.42^{* *}$ & $-.39^{* *}$ & $.18^{* *}$ & $-.29^{* *}$ & $.60^{* *}$ & - & & & \\
\hline 10. Mental Health & 2.79 & 1.06 & $.67^{* *}$ & $-.64^{* *}$ & $-.11^{* *}$ & $.38^{* *}$ & $-.44^{* *}$ & $.25^{* *}$ & $-.58^{* *}$ & $.70^{* *}$ & $.53^{* *}$ & & & \\
\hline \multicolumn{15}{|l|}{ Mindsets } \\
\hline 11. Catastrophe & 4.78 & 1.08 & $-.17^{* *}$ & $.24^{* *}$ & $.29^{* *}$ & -.02 & $.15^{* *}$ & -.01 & $.21^{* *}$ & $-.16^{* *}$ & $-.10^{* *}$ & $-.17^{* *}$ & - & \\
\hline 12. Manageable & 3.66 & 1.34 & $.17^{* *}$ & $-.16^{* *}$ & $-.31^{* *}$ & $.04^{* *}$ & $-.11^{* *}$ & .00 & $-.14^{* *}$ & $.16^{* *}$ & $.12^{* *}$ & $.15^{* *}$ & $-.17^{* *}$ & - \\
\hline 13. Opportunity & 4.96 & 1.02 & $.06^{* *}$ & $.08^{* *}$ & $.33^{* *}$ & $.09^{* *}$ & $.07^{* *}$ & $.21^{* *}$ & .02 & $.04^{* *}$ & .00 & -.02 & $.20^{* *}$ & $-.07^{* *}$ \\
\hline
\end{tabular}

Table S4. Correlations between measures of affect, behaviors, wellbeing, and mindsets measured at T3 (September $16^{\text {th }}-27^{\text {th }}, 2020$ ). M and SD indicate mean and standard deviation, respectively. Asterisks indicate significance of Pearson's correlations such that " $\mathrm{p} \leq 0.01$ and ${ }^{* *} \mathrm{p} \leq 0.001$. Cronbach's alphas are included in parentheses on the diagonal for summary scores (but not total scores or individual items). 


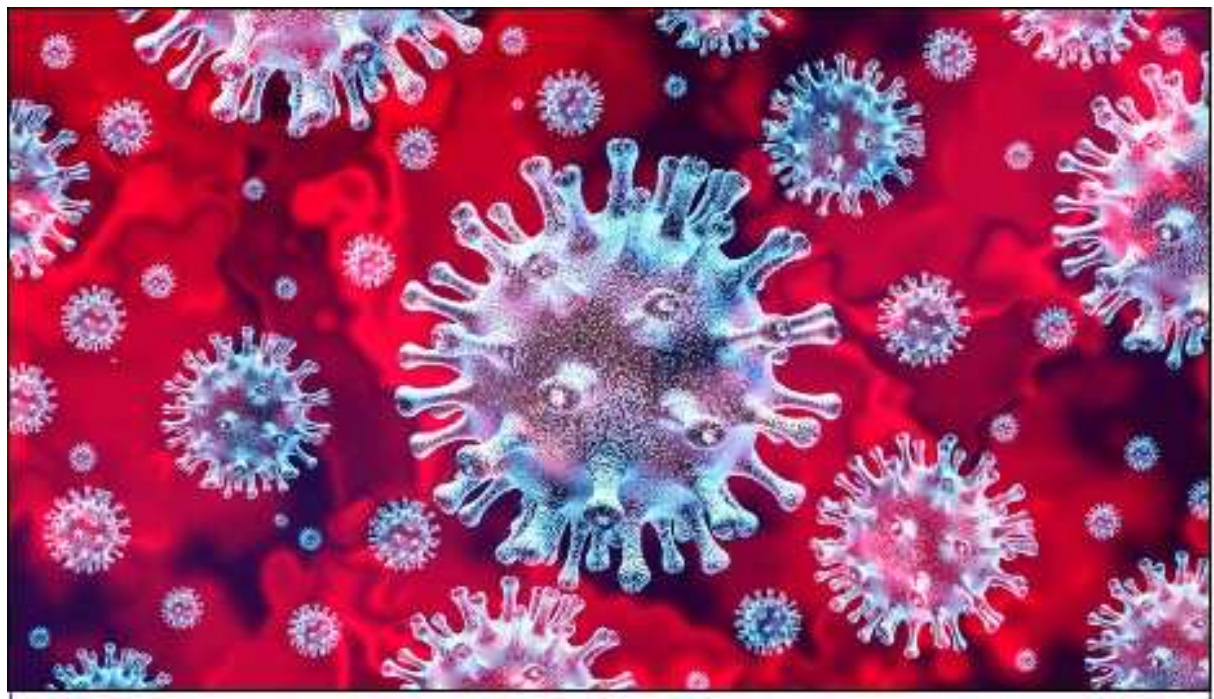

Stanford researchers are interested in American residents' perceptions of and reactions to the COVID-19 coronavirus. Complete a 15-20 minute survey, enter a draw to win 1 of $10 \$ 100$ Amazon Gift Cards, and have your say!

Figure S1. Social media advertisement posted on Facebook and Twitter for 10 days beginning March 11th, 2020. Clicking on the advertisement directed interested participants to a Qualtrics survey. 
(a)
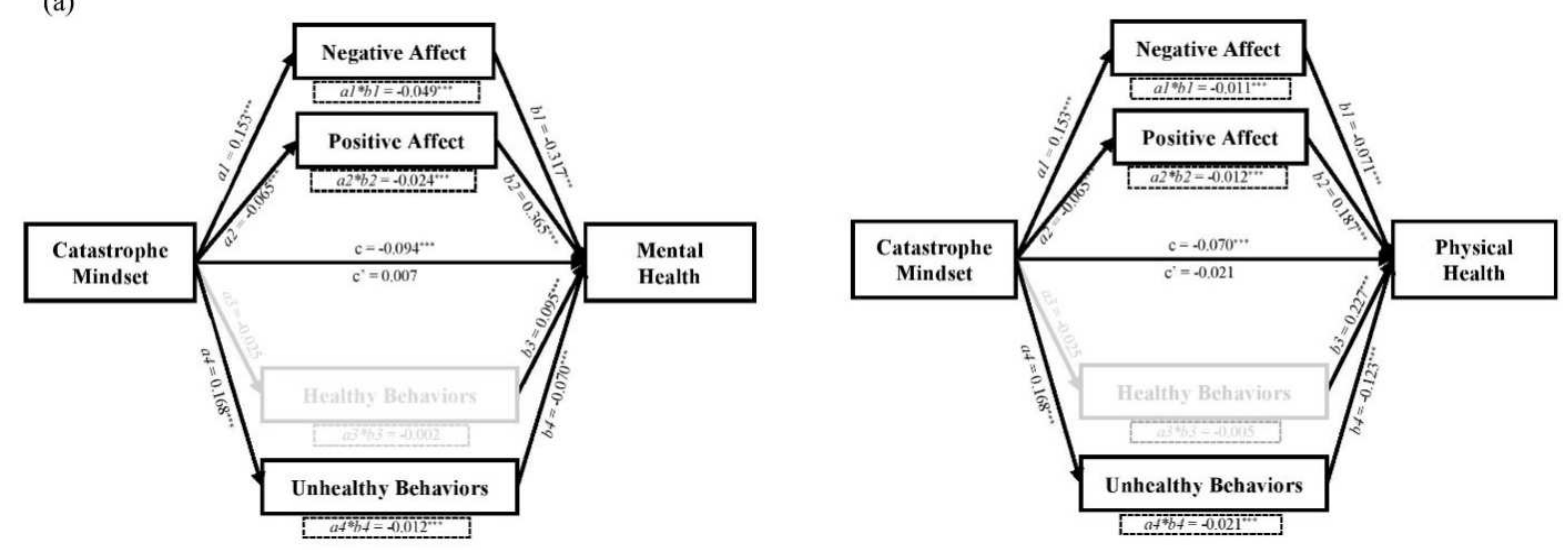

(b)
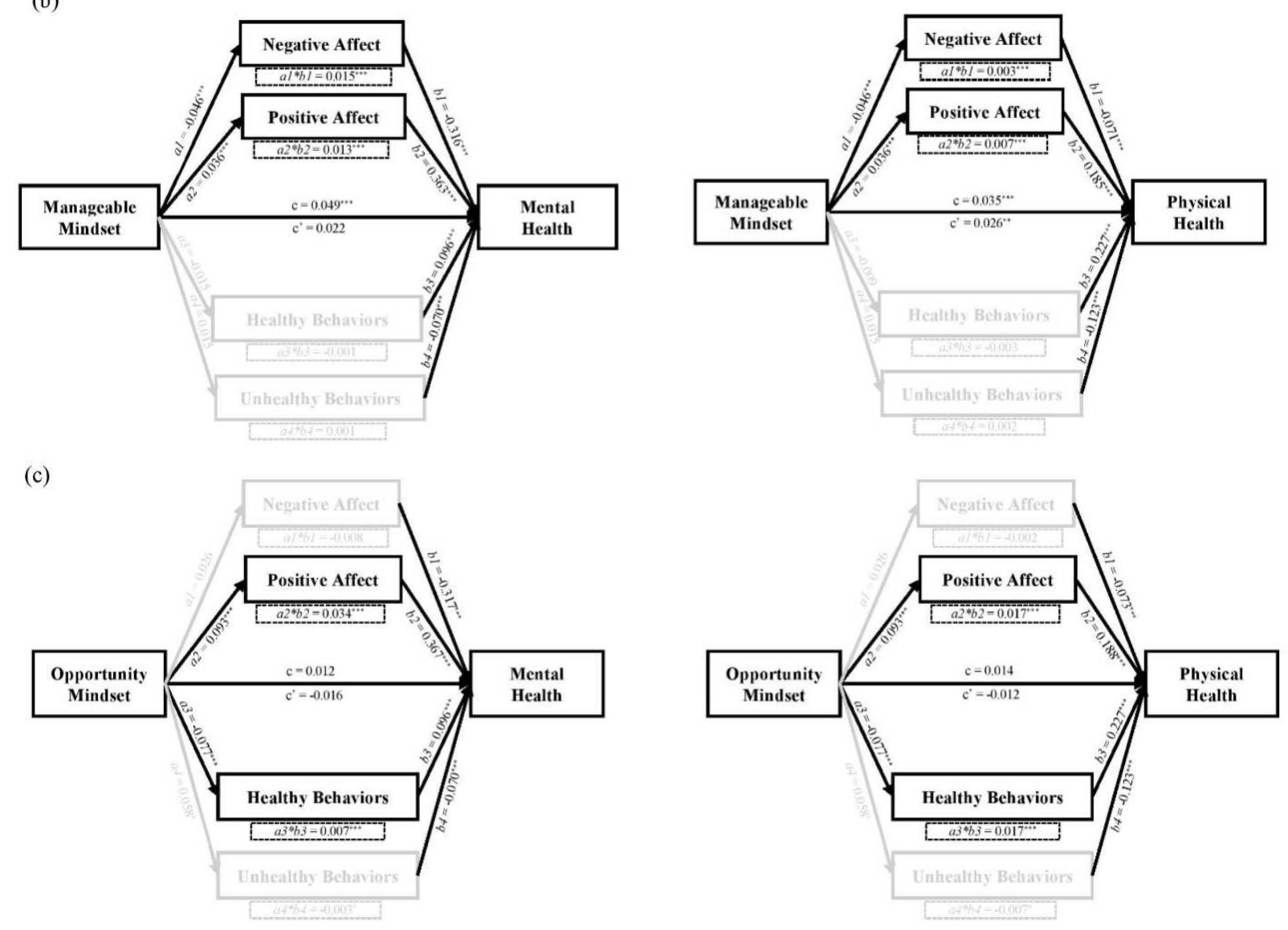

Figure S2. Mediation models outlining the mechanisms through which the (a) catastrophe mindset, (b) manageable mindset, and (c) opportunity mindset influence mental and physical health.

Mindsets were measured at T1 (March 2020), affective and behavioral mediators were measured at T2 (May 2020), and health outcomes were measured at T3 (September 2020). 
Table S5. Baseline demographics across retention.

\begin{tabular}{|c|c|c|c|c|}
\hline & $\begin{array}{l}\text { Included } \\
(\mathrm{N}=5365)\end{array}$ & $\begin{array}{l}\text { Not Included } \\
(\mathrm{N}=14417)\end{array}$ & $\begin{array}{l}\text { Total } \\
(\mathrm{N}=19782)\end{array}$ & $p$ value \\
\hline Age & & & & $<0.001$ \\
\hline $18-24$ & $261(4.9 \%)$ & $879(6.1 \%)$ & $1140(5.8 \%)$ & \\
\hline $25-44$ & $2529(47.1 \%)$ & $7227(50.3 \%)$ & $9756(49.5 \%)$ & \\
\hline $45-64$ & $1915(35.7 \%)$ & $5032(35.1 \%)$ & $6947(35.2 \%)$ & \\
\hline $65+$ & $660(12.3 \%)$ & $1217(8.5 \%)$ & $1877(9.5 \%)$ & \\
\hline Gender & & & & $<0.001$ \\
\hline Man & $1008(18.8 \%)$ & $3459(24.4 \%)$ & $4467(22.9 \%)$ & \\
\hline Woman & $4357(81.2 \%)$ & $10707(75.6 \%)$ & $15064(77.1 \%)$ & \\
\hline Education & & & & $<0.001$ \\
\hline Bachelor's & $4310(80.3 \%)$ & $9706(67.7 \%)$ & $14016(71.2 \%)$ & \\
\hline No Bachelor's & $1055(19.7 \%)$ & $4627(32.3 \%)$ & $5682(28.8 \%)$ & \\
\hline Race & & & & $<0.001$ \\
\hline Non-White & $575(10.7 \%)$ & $1793(12.5 \%)$ & $2368(12.0 \%)$ & \\
\hline White & $4790(89.3 \%)$ & $12546(87.5 \%)$ & $17336(88.0 \%)$ & \\
\hline Political Affiliation & & & & $<0.001$ \\
\hline Democrat & $3019(56.3 \%)$ & $6143(43.1 \%)$ & $9162(46.7 \%)$ & \\
\hline Indep/Other & $1663(31.0 \%)$ & $5115(35.9 \%)$ & $6778(34.6 \%)$ & \\
\hline Republican & $683(12.7 \%)$ & $2981(20.9 \%)$ & $3664(18.7 \%)$ & \\
\hline
\end{tabular}


Table S6. Baseline mindsets across retention.

\begin{tabular}{lllll}
\hline & $\begin{array}{r}\text { Included } \\
\mathbf{( N = 5 3 6 5 )}\end{array}$ & $\begin{array}{c}\text { Not Included } \\
(\mathbf{N = 1 4 4 1 7 )}\end{array}$ & $\begin{array}{c}\text { Total } \\
(\mathbf{N = 1 9 7 8 2})\end{array}$ & p value \\
\cline { 2 - 5 } Catastrophe Mindset & $4.80(1.17)$ & $4.71(1.24)$ & $4.74(1.22)$ & $<0.001$ \\
Mean (SD) & & & & $<0.001$ \\
\hline Manageable Mindset & $2.67(1.46)$ & $2.97(1.54)$ & $2.89(1.52)$ & \\
Mean (SD) & & & & $<0.001$ \\
\hline Opportunity Mindset & $5.06(0.99)$ & $4.95(1.08)$ & $4.98(1.06)$ & \\
Mean (SD) &
\end{tabular}

Table S7. Baseline outcome variables across retention.

\begin{tabular}{|c|c|c|c|c|}
\hline & $\begin{array}{l}\text { Included } \\
(\mathrm{N}=5365)\end{array}$ & $\begin{array}{c}\text { Not Included } \\
(\mathrm{N}=14417)\end{array}$ & $\begin{array}{c}\text { Total } \\
(\mathrm{N}=19782)\end{array}$ & p value \\
\hline CDC Behaviors & & & & $<0.001$ \\
\hline Mean (SD) & $3.56(0.55)$ & $3.52(0.63$ & $3.53(0.61)$ & \\
\hline Healthy Behaviors & & & & $<0.001$ \\
\hline Mean (SD) & $3.32(0.86)$ & $3.20(0.87)$ & $3.23(0.87)$ & \\
\hline Positive Affect & & & & 0.049 \\
\hline Mean (SD) & $2.80(0.77)$ & $2.82(0.82)$ & $2.82(0.80)$ & \\
\hline Negative Affect & & & & 0.788 \\
\hline Mean (SD) & $2.61(0.92)$ & $2.60(0.97)$ & $2.60(0.96)$ & \\
\hline Quality of Life & & & & 0.001 \\
\hline Mean (SD) & $3.28(0.99)$ & $3.23(1.02)$ & $3.24(1.01)$ & \\
\hline Physical Health & & & & $<0.001$ \\
\hline Mean (SD) & $3.42(0.97)$ & $3.33(0.97)$ & $3.36(0.97)$ & \\
\hline
\end{tabular}

